\title{
Human papillomavirus in head and neck cancer
}

\section{David A. Palma MD PhD, Anthony C. Nichols MD \\ The incidence of head and neck cancers related to human papillomavirus is rising}

In the 1980s, human papillomavirus (HPV) was found in less than $20 \%$ of oropharyngeal cancers in the United States, but this proportion increased to $70 \%$ by 2000-2004. ${ }^{1}$ These cancers are usually found in the tonsils or at the base of the tongue. The annual incidence of HPV-related oropharyngeal tumours increased by $225 \%$ over a similar period; by 2020 , it will surpass the incidence of cervical cancer. ${ }^{1}$ In contrast, the incidence of head and neck cancers related to smoking and alcohol is declining. ${ }^{1}$

Head and neck cancers related to HPV occur earlier than typical oropharyngeal cancers

Most patients with HPV-related cancers of the head and neck are 50 to 60 years old, 10 years younger than typical patients with oropharyngeal cancer. Risk factors for HPV-related cancers include higher lifetime number of sexual partners, higher number of oral sex partners and marijuana use. ${ }^{2}$ Human papillomavirus subtype 16 is responsible for about $90 \%$ of cases; ${ }^{1}$ it is also the most common subtype found in cervical cancer.

\section{Cancers related to HPV are a distinct disease entity from smoking-related cancers}

Patients with HPV-related cancers are often nonsmokers. Human papillomavirus oncogenes E6 and E7 degrade or inactivate cell-cycle regulators p53 and pRb, whereas smoking-related cancers are driven by mutations in tumour suppressor genes. The HPV status in tumour tissue is commonly ascertained using immunohis tochemistry for p16 protein (Figure 1).

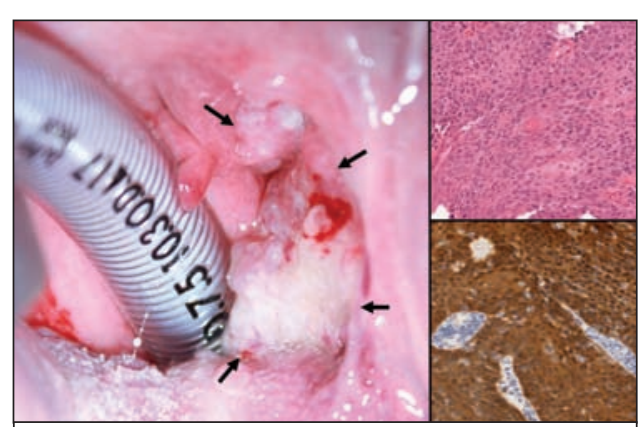

Figure 1: Tumours related to human papillomavirus (HPV) are usually found in the tonsil (left panel, arrows) or base of the tongue, and are of squamous cell histology (upper right panel). Immunohistochemistry for p16 protein (lower right panel, brown) is a surrogate for HPV.

For references, please see Appendix 1, available at www.cmaj.ca/lookup /suppl/doi:10.1503/cmaj.130849/-/DC1

Survival outcomes for HPV-related cancers are very good, in contrast to smoking-related head and neck cancers

Cancers of the head and neck related to HPV are commonly treated with radiotherapy and concurrent cisplatin, with 3 -year survival of greater than $80 \% .^{3}$ However, such treatment can be associated with substantial long-term adverse sequelae, including swallowing dysfunction and feeding-tube dependence. Randomized trials are assessing strategies for treatment de-escalation, including substituting epidermal growth factor receptor inhibitors for cisplatin, or primary transoral robotic surgery instead of radiotherapy. ${ }^{4}$

Head and neck cancers related to HPV are likely preventable with vaccination

A randomized trial showed that HPV vaccination reduces rates of oropharyngeal HPV infection by more than $90 \% .^{5}$ This will likely translate into a reduced incidence of HPV-related oropharyngeal cancers in the future.

Competing interests: Anthony Nichols has received a grant from Merck Canada for research on HPV-related head and neck cancer. No competing interests were declared by David Palma.

This article has been peer reviewed.

Affiliations: Department of Otolaryngology Head and Neck Surgery (Palma, Nichols); Division of Radiation Oncology (Palma), Department of Oncology, London Health Sciences Centre and Western University, London, Ont.

Correspondence to: David Palma, david.palma @1hsc.on.ca

CMAJ 2014. DOI:10.1503/cmaj.130849 